Rhode Island College

Digital Commons @ RIC

\title{
The Impact of Rhode Island's Mandatory Health Care Worker Influenza Vaccination Regulation on Influenza Incidence in Long- Term Care Facility Residents
}

Wendy A. Chicoine

Follow this and additional works at: https://digitalcommons.ric.edu/etd

Part of the Occupational and Environmental Health Nursing Commons

\section{Recommended Citation}

Chicoine, Wendy A., "The Impact of Rhode Island's Mandatory Health Care Worker Influenza Vaccination Regulation on Influenza Incidence in Long-Term Care Facility Residents" (2018). Master's Theses, Dissertations, Graduate Research and Major Papers Overview. 262.

https://digitalcommons.ric.edu/etd/262

This Thesis is brought to you for free and open access by the Master's Theses, Dissertations, Graduate Research and Major Papers at Digital Commons @ RIC. It has been accepted for inclusion in Master's Theses, Dissertations, Graduate Research and Major Papers Overview by an authorized administrator of Digital Commons @ RIC. For more information, please contact digitalcommons@ric.edu. 
THE IMPACT OF RHODE ISLAND'S MANDATORY HEALTH CARE WORKER INFLUENZA VACCINATION REGULATION ON INFLUENZA INCIDENCE IN

\section{LONG-TERM CARE FACILITY RESIDENTS}

by

Wendy A. Chicoine

A Major Paper Submitted in Partial Fulfillment

of the Requirements for the Degree of

Master of Science in Nursing

in

The School of Nursing

Rhode Island College

2018 


\begin{abstract}
Seasonal influenza is a serious public health problem that contributes to significant morbidity and mortality locally, nationally, and globally. The Centers for Disease Control and Prevention (CDC) estimates that influenza has caused between 9.2 million and 60.8 million illnesses, between 140,000 and 710,000 hospitalizations, and between 12,000 and 56,000 deaths annually since 2010, and approximately $80-90$ percent of influenza related deaths occurred in people 65 years and older. In 2012, prompted by the mounting evidence of the risk of nosocomial influenza infection for patients and low influenza vaccination rates of health care workers, the Rhode Island Department of Health (RIDOH) amended its Rules and Regulations for Immunization and Testing for Healthcare Workers. This amendment included mandatory influenza vaccinations for all health care workers, students, volunteers, and trainees who have direct patient contact within a health care facility. The purpose of this study was to evaluate the impact of the regulation of mandating influenza vaccination for health care workers in Rhode Island on influenza incidence, hospitalizations, and deaths of residents of long-term care facilities (LTCF). Surveillance data for influenza incidence, hospitalizations, and deaths among residents of LTCF pre and post the policy implementation are analyzed and results presented. Policy and practice implications for public health nursing are discussed.
\end{abstract}




\section{Acknowledgements}

First, I would like to thank my thesis advisor and first reader Dr. Joanne Costello of the school of nursing at Rhode Island College. I would like to acknowledge Dr. Costello for her expertise and guidance throughout this journey. Without her encouragement and support, this work would not have been possible. Dr. Costello's positivity and inspiration allowed me to produce my best work. I would also like to thank Esperanza Gutierrez also of the School of Nursing at Rhode Island College as my second reader. I am grateful for her very valuable comments on this thesis. I would also like to recognize Diane Brady from the Rhode Island Department of Health as my third reader. Diane has been instrumental in my success in completing this project. I am eternally grateful for her input and fortunate to have had the opportunity to work with her on this project. Many other individuals including, Rhode Island College's faculty member Wendy Doremus, Rhode Island Department of Health's Tricia Washburn, and Annie Gjelsvik from Brown University School of Public Health have also been valuable resources for this project, and I would like to thank them for their contribution.

Finally, I must express my very profound gratitude to my family, especially my husband Michael, and my three children, Taylor, Paige, and Christian for providing me with unfailing support and continuous encouragement and patience throughout my years of study. This accomplishment would not have been possible without them. 


\section{Table of Contents}

Background/Statement of the Problem ............................................................... 1

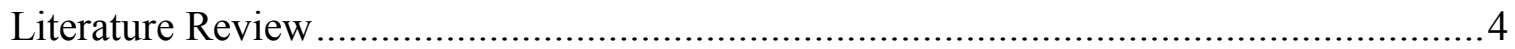

Theoretical Framework ................................................................................... 20

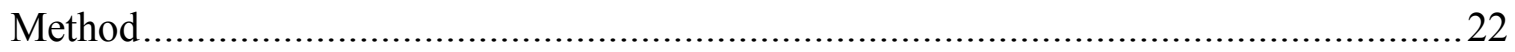

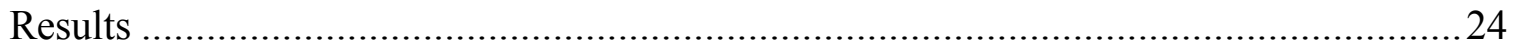

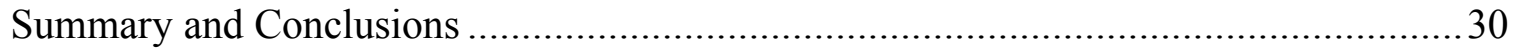

Recommendations and Implications for Advanced Nursing Practice......................... 32

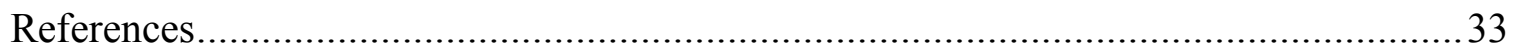

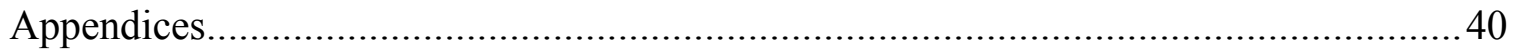


The Impact of Rhode Island's Mandatory Health Care Worker Influenza Vaccination Regulation on Influenza Incidence in Long-Term Care Facility Residents

\section{Background/Statement of the Problem}

Seasonal influenza is a serious public health problem that contributes to significant morbidity and mortality locally, nationally, and globally (Centers for Disease Control and Prevention [CDC], 2016d). It is an acute viral infection that occurs every year, peaks during the winter months, occurs worldwide, and can affect any age group. The three types of influenza viruses that cause human disease are influenza A, B and C. Each of these viruses may be categorized into subtypes according to the combinations of various virus surface proteins. Among the different subtypes, influenza A (H1N1) and $(\mathrm{H} 3 \mathrm{~N} 2)$ are the most common among humans. Influenza B viruses are usually only found in humans (and seal populations) and are less severe than influenza type A. Influenza type $\mathrm{C}$ typically causes a mild respiratory illness and generally does not cause epidemics (CDC, 2016d).

Influenza affects all populations. However, children younger than two, adults aged 65 and older, pregnant women, and those with certain medical conditions, including chronic heart, lung, kidney, liver, blood or metabolic diseases such as diabetes or a weakened immune system are most at risk (CDC, 2016d). Individuals living in long-term care facilities (LTCF) are also considered to be at high risk since many individuals of advanced age and with multiple chronic health conditions reside in these facilities. In addition, congregate living situations provide an environment facilitating the spread of infectious disease. The Centers of Disease Control and Prevention (CDC) estimates that approximately 80-90 percent of influenza related deaths occurred in people 65 years and 
older (CDC 2017a). Understanding the history of influenza, the importance of prevention, and knowing the signs and symptoms of the disease are very important factors in controlling the spread of the virus and decreasing serious illness and deaths caused by it.

Prompted by the mounting evidence of the risk of nosocomial influenza infection for patients and low influenza vaccination rates of health care workers, the Rhode Island Department of Health (RIDOH) amended its Rules and Regulations for Immunization and Testing for Healthcare Workers in 2012 (Keough, 2014). During the 2011-2012 influenza season, only $69.7 \%$ of health care workers were vaccinated against influenza (Kim, Raymond, Washburn, \& Capelli, 2014). This amendment included mandatory influenza vaccinations for all health care workers, students, volunteers, and trainees who have direct patient contact within a health care facility (State of Rhode Island and Providence Plantations Department of Health, 2012). Dr. Michael Fine, Director of the RIDOH during this time, determined that it was a health care quality and safety issue and therefore the duty of those who worked in health care to protect the individuals for whom they provided direct care (Rhode Island Department of Health [RIDOH], 2012). He initiated the regulatory change based upon patient safety goals as a strategy to prevent the spread of influenza to our most vulnerable population through mandatory influenza vaccination (RIDOH, 2012).

The purpose of this study was to evaluate the impact of the regulation of mandatory influenza vaccination for health care workers in the State of Rhode Island on influenza incidence in LTCF. The rationale for this study was to explore the benefit of the state mandate for vulnerable populations, in particular for residents in LTCF. 
Secondary data was analyzed to evaluate the impact of the RIDOH public health intervention. 


\section{Literature Review}

A comprehensive literature review was conducted utilizing online databases including: state and federal government websites, CINAHL, JSTOR, Medline Plus, PubMed, and Cochrane Library. Key search words included: influenza, nosocomial infection influenza, influenza prevention, health care worker vaccination, influenza vaccine and patient safety, mandatory influenza, and health care worker vaccination effects on patient safety. This literature search included references from 1997 through 2017.

\section{Influenza Virus: Scope and Burden of Disease}

The influenza virus is transmitted from person to person primarily through respiratory droplets. When an infected person coughs or sneezes within close contact of another, the virus can be spread. These droplets do not remain suspended in the air and can only travel short distances. However, indirect transmission through contact with these droplets on contaminated surfaces provides another source of transmission (CDC, 2016d). The incubation period for influenza is one to four days, with an average of two days. A healthy adult could infect others beginning one day before they are symptomatic and up to five to seven days after becoming ill (CDC, 2016d). Young children and those who are immunocompromised may be able to infect others for a longer period of time (CDC, 2016d).

Influenza that is uncomplicated has an onset of fever, myalgia, headache, malaise, nonproductive cough, sore throat, and rhinitis (CDC, 2016d). Without complications, typically the influenza will resolve after three to seven days for most people. However, the cough and malaise can continue for greater than two weeks. The influenza virus can 
cause primary influenza viral pneumonia and can exacerbate underlying health conditions, such as cardiac and pulmonary diseases. This can lead to secondary bacterial pneumonia, sinusitis, or otitis media (CDC, 2016d). In the United States, influenza epidemics tend to occur during the fall and winter months, however can peak as late as April or May. Illness can result in hospitalizations and death (CDC, 2016d).

The precise number of deaths caused by the influenza virus is difficult to track, and the rates of disease may therefore be underreported. Death certificates may not list influenza as the primary cause of death, and states are not required to report influenza cases or deaths of individuals older than 18 years old to the CDC. Most of the flu-related deaths occur one to two weeks after the initial infection, which may lead to secondary infections or exacerbation of existing conditions.

The Epidemiology and Prevention Branch in the influenza division of the CDC analyzes data collected to determine the influenza virus that is circulating and when and where activity is occurring. It tracks influenza like illness (ILI) and measures the impact on deaths and hospitalizations to detect changes in influenza viruses. This data collected throughout the year on influenza activity is from state and local health departments, public health and clinical laboratories, vital statistics offices, health care providers, clinics, and emergency departments (CDC, 2016c). Using this surveillance data, the CDC reports that an estimated 9.2 -35.6 million experienced illness, 140,000 - 710,000 were hospitalized, and 12,000 - 56,000 died of influenza related causes (CDC, 2017a). 


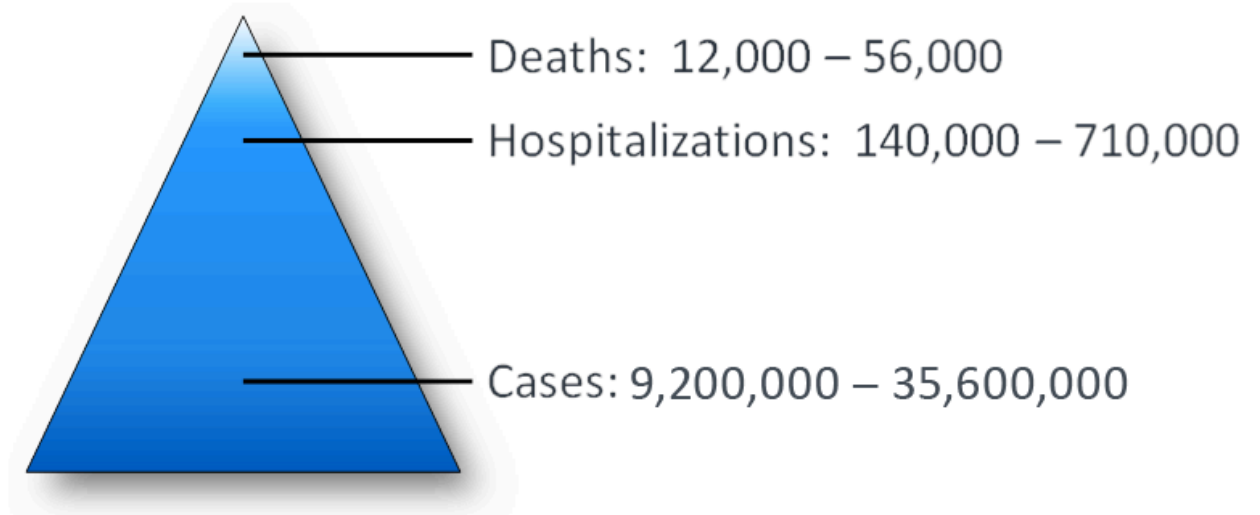

Figure 1. Influenza Disease in the United States Annually Since 2010 (CDC, 2017a).

The burden of influenza disease is significant in terms of morbidity and mortality, but other more indirect burdens also exist. Molinari et al. (2007) evaluated the annual impact of seasonal influenza in the United States. Based on the United States population in 2003, results of this study indicated an estimate of annual influenza epidemics resulted in an average of 610,660 life-years lost, 3.1 million hospitalized days, and 31.4 million outpatient visits, with an average of $\$ 10.4$ billion dollars in direct costs (Molinari et al., 2007). The authors also noted estimated loss of wages due to illness and loss of life amounted to $\$ 16.3$ billion dollars annually. The total economic burden amounted to \$87.1 billion dollars annually (Molinari et al., 2007). It is clear that influenza has a major impact on the health of our population.

Influenza surveillance in Rhode Island is maintained by the Center for Acute Infectious Disease Epidemiology (CAIDE). Each influenza season, the RIDOH provides a surveillance summary develop by CAIDE. The data collected by CAIDE include hospitalizations and deaths, which is used to monitor and assess the intensity of influenza and the spread of ILI within the community (Burns et al., 2017). This information is used to provide a complete depiction of influenza in the state of Rhode Island (Burns et al., 
2017). In Rhode Island, for the 2016-2017 season, 5,045 influenza specimens tested positive at Rhode Island hospitals. Of the 5,045 who had tested positive, 1,216 individuals were hospitalized and 33 adult influenza related deaths occurred (Burns et al., 2017). In the 2016-2017 influenza season, most deaths occurred in adults 65 years and older.

Individuals in LTCF whose immunity is frequently compromised due to advanced age or illness are a population of significant concern. This population is susceptible to complications as well as nosocomial infections. Primary prevention through vaccination of those who are most vulnerable as well as vaccination of health care workers is essential in preventing the spread of influenza and reducing nosocomial influenza infections (CDC, 2017c).

\section{Nosocomial Influenza Infection}

A nosocomial infection, or hospital acquired infection/health care acquired infection (HAI), is an infection that is transmitted in a hospital or health care facility. Infections occurring more than 48 hours after admission, three days after discharge, or 30 days after surgery are considered nosocomial (Inweregbu, Dave, \& Pettard, 2005). Nosocomial influenza infections in acute and long-term hospital facilities were described by Eibach et al. (2014) as a life-threatening risk for geriatric patients who may be nosocomially infected by health care workers, other patients, or visitors. The aim of this study was to understand how influenza is transmitted and to identify measures of prevention. The authors described routes of transmission of influenza in geriatric patients and health care workers at a hospital in France. All patients and health care workers of the acute care geriatric floor were screened for ILI occurring during the peak of the 
2011/2012 influenza season. For this study, symptoms that were reported at least 72 hours after admission would be categorized as nosocomial. A total of 66 patients and 57 health care workers were screened for influenza during the study period. Of this sample, 16 patients and six health care workers tested positive for influenza A. The mean time from admission to onset of symptoms was 23.2 days. Six patients with an average age of 88.3 years had acquired influenza infection 72 hours after admission, which classified this as a nosocomial influenza infection (Eibach et al., 2014).

This study highlighted the possibility that health care workers introduced the influenza virus to the hospital based on molecular characteristics of the strains identified in these outbreaks (Eibach et al., 2014). The authors were able to identify several outbreaks within the hospital, rather than one, by using an epidemiologic and molecular approach (Eibach et al., 2014). Transmission of influenza from health care workers to patients is common and despite annual health care worker influenza vaccination recommendations, both on a local and national level, health care worker vaccination rates remained low in this facility (Eibach et al., 2014).

Factors such as vaccine effectiveness among the elderly, vaccine mismatch during this influenza season, and vaccine effectiveness during the late influenza season, due to changes in the virus and waning immunity were considered potential contributors to the outbreaks. Based upon the results, the authors confirmed that health care workers transmitted the influenza virus to their patients. Recommendations for practice were "higher vaccination rates, isolation measures and better hand hygiene to prevent future outbreaks" (Eibach et al., 2014, p.188). 
Another study by Frenzel et al. (2016) explored a relationship between increased influenza vaccinations in health care workers with a reduction of nosocomial infections in cancer patients within an inpatient cancer center in Texas. The authors described influenza vaccination of health care workers as a "core patient and health care worker safety practice and its association with preventing nosocomial infection of influenza and reduction of patient mortality" (Frenzel et al., 2016, p.1016). This eight-year study began in 2006 with strategies such as educational staff in-services and on-site staff vaccination clinics.

In 2009, a pilot was developed that mandated participation in an influenza prevention program which focused on vaccination of health care workers who work in high-risk areas. In 2011, Texas passed a law requiring health care facilities to implement a vaccine-preventable disease policy. This enabled facilities to implement a policy for mandatory vaccination programs that would require all health care workers to receive influenza vaccination or to wear a surgical mask when providing patient care during influenza seasons (Frenzel et al., 2016). The researchers observed that the new mandate not only improved the annual influenza vaccination rates from $56 \%$ in $2006-2007$ to $94 \%$ in 2013-2014, but was also associated with a decrease in nosocomial infections in cancer patients (Frenzel et al., 2016).

\section{Influenza Prevention}

The most effective way to prevent influenza infection is through vaccination (CDC, 2016d). Vaccines are known as one of the greatest public health achievements and have significantly decreased the morbidity and mortality rates of infectious diseases. Edward Jenner and his contribution to immunizations and the eradication of smallpox 
established the foundation of vaccination as a safe and effective tool to prevent infectious disease in populations, shifting leading causes of death to chronic disease in the last century (College of Physicians of Philadelphia, 2018). The Advisory Committee on Immunization Practices (ACIP) provides advice and recommendations to the CDC regarding use of vaccines for vaccine preventable diseases (CDC, 2017e). These recommendations are reviewed by the $\mathrm{CDC}$ director, and if approved, are published as official CDC recommendation in the Morbidity and Mortality Weekly Report (MMWR) (CDC, 2017e).

The ACIP also provides vaccination recommendations for health care workers. Vaccines included in these recommendations are hepatitis B, mumps, measles and rubella (MMR), varicella, Tdap, meningococcal, and influenza (CDC, 2011b). In addition to the ACIP recommendations for vaccination of health care workers, laws exist regarding vaccination requirements for particular populations. Included in these laws are vaccination requirements for children in public and private schools and daycare settings, college/university students, health care workers, and patients in LTCF (CDC, 2017d). These laws provide health care facilities with ability to establish these requirements according to mandates in state statutes and regulations. In 2012, Rhode Island included influenza immunization as a requirement within their Rules and Regulations Pertaining to Healthcare Workers (State of Rhode Island and Providence Plantations Department of Health, 2012).

\section{Influenza Vaccine and Health Care Workers}

The CDC estimates between $80 \%$ and $90 \%$ of influenza related deaths occurred in people 65 years and older. During the 2012-2013 influenza season, a total of 12,336 
influenza associated hospitalizations were reported in the U.S. (CDC, 2017a). People 65 years and older accounted for approximately half of these hospitalizations. Health care workers who are not vaccinated may become infected through contact with an infected resident, visitors, family members or an infected health care worker and may spread influenza to residents and others. The CDC provides recommendations for health care worker vaccination, which includes influenza vaccine, with a goal of reducing the spread of influenza infection, especially for patients who are most vulnerable. Guidance is also provided for health care workers working in long-term care. Influenza infection in older adults and individuals living with chronic medical conditions poses a serious health threat. Older individuals typically have decreased immune response to the influenza vaccine; decreased functionality of the immune systems, combined with chronic medical conditions, increases the likelihood of poor health outcomes related to influenza virus (CDC, 2017c). Therefore, it is important for those who care for high-risk individuals, especially those who work in LTCF, to receive annual influenza vaccination (CDC, 2017c). Vaccination is the most effective intervention to prevent the spread of influenza. Influenza vaccination coverage among health care personnel for the 2015-2016 influenza seasons was published in the MMWR (CDC, 2016b). Coverage was noted to be higher among health care personnel working in the hospital setting $(91.2 \%)$, lower in ambulatory care settings (79.8\%), and the lowest in long-term care settings (69.2\%). Although the lowest vaccination rate was reported in long-term care settings, this rate was an increase from $63.9 \%$ in the previous 2014-2015-influenza season (CDC, 2016b). Vaccination coverage was noted to be lowest in settings where influenza vaccination was not required, encouraged, or offered on-site. Implications for practice presented were 
employer vaccination requirements, on-site vaccination at no cost, and implementing evidence-based intervention strategies to address reasons why health care workers decline vaccination (CDC, 2016b).

An article by Pless, McLennan, Nicca, and Elger (2017) explored reasons why nurses decline influenza vaccine. Methods for this study included individual semistructured interviews with 18 nurses from a variety of settings and positions. In this qualitative study, the authors revealed three themes explaining why nurses decline influenza vaccination. These reasons were: maintaining a strong and healthy body; maintaining autonomy; and being in an untrustworthy environment.

Perceptions of vaccine efficacy and side effects fell under the category of maintaining a healthy body. Nurses stated they did not believe influenza posed a threat since they were healthy. They also reported fear of side effects, feeling ill or observing others feeling ill after receiving the vaccine in the past as a reason for declining vaccination. Maintaining autonomy was especially important and their ability to make their own choices regarding influenza vaccination. Nurses also feared that accepting influenza vaccination would open the door for more initiatives that would restrict their autonomy (Pless, McLennan, Nicca, \& Elger, 2017). The last theme was lack of trust. Lack of efficacy and safety of influenza vaccine had an impact on the nurses' decision to receive vaccination. Some nurses disputed that the number of influenza viruses and the frequent mutation of the virus, only makes the vaccine an assumption and therefore not effective. They also did not believe the vaccine was safe due to the pace needed for annual production along with doubts regarding true motivations of those who are promoting the vaccine (Pless et al., 2017). 
The authors noted in their interviews lack of awareness among nurses as to the importance of influenza vaccination to protect vulnerable patients (Pless et al., 2017). The authors concluded interventions to increase influenza vaccination among nurses should include training that empowers nurses to make decisions based on evidence and should be integrated into nursing programs, the workplace, and continuing education programs (Pless et al., 2017).

A qualitative study by Willis and Wortley (2007) also assessed nurses' attitudes and beliefs regarding influenza and influenza vaccine. Just as Pless et al. (2017) noted, Willis and Wortley (2007) also identified that a contributing factor for the low uptake of influenza vaccination among nurses was associated with concerns about influenza efficacy and safety. The authors cited availability of vaccination administration as another factor. In addition, the authors noted lack of education regarding the efficacy and safety of influenza vaccine played a role in promoting vaccination to their patients (Willis $\&$ Wortley, 2007). Many nurses were not aware of the rationales for health care worker vaccination and their role in reducing influenza transmission to patients. Limitations of this study cited by the authors included a small sample size of vaccinated $(\mathrm{N}=34)$ and unvaccinated ( $\mathrm{N}=37$ ) $\mathrm{RN}$ 's and the sample being from only two locations (Birmingham, Alabama and Detroit, Michigan). Willis and Wortley (2007) concluded that there is no single approach that will increase vaccination rates. Recommendations for practice were educational programs to include rationales for vaccination and making receipt of vaccinations convenient.

In response to low health care workers influenza vaccination rates in Rhode Island, in particular a rate of 33.9\% among workers in LTCF, Marshall, Tetu-Mouradjian, 
\& Fulton (2010) aimed to assess the attitude, values and beliefs associated with health care workers who did not receive influenza vaccine during the 2006-2007 season. Using the stages of change theory and the social marketing approach, the authors explored ways to increase influenza vaccination among health care workers (Marshall et al., 2010). The six stages of change include: pre-contemplation, contemplation, preparation, action, maintenance and relapse. Marshall et al. (2010) focused on two categories, precontemplation versus maintenance with respondents in the maintenance stage representing the ideal state and respondents in the pre-contemplative stage representing those least likely to adapt to the ideal state.

A convenience sample of 846 registered nurses, licensed practical nurses, and certified nursing assistants were surveyed about influenza vaccine. The survey included questions related to influenza vaccination history, perceptions of influenza virus, severity of influenza virus, and possible complications for oneself and others, the cost and benefits of influenza vaccine as well as influenza vaccine in relation to other preventative measures such as hand hygiene and other vaccines. The participants were also asked to comment on circumstances that might increase their likelihood of receiving influenza vaccination the following influenza season (Marshall et al., 2010). Health care workers in the pre-contemplative stage perceived influenza virus as low risk and ineffective, and they reported that the influenza vaccine made them ill. However, given the right circumstances, such as developing a high risk chronic illness or having an immunocompromised family member, they reported being more likely to get vaccinated for influenza (Marshall et al., 2010). Health care workers in the maintenance stage 
reported influenza vaccine as useful and were likely to support vaccine mandates for health care workers (Marshall et al., 2010).

Results of this study suggest a policy requiring influenza vaccination of health care workers would move individuals from pre-contemplation to action and then to the maintenance stage of behavior change (Marshall et al., 2010). This behavior change could have a positive impact on public health and patient safety by increasing the number of health care workers who receive influenza vaccination.

\section{Mandatory Influenza Vaccination of Health Care Workers and Patient Safety}

In an effort to increase influenza vaccination coverage among health care workers and to attempt to meet the Healthy People 2020 objective of $90 \%$ health care worker vaccination (Office of Disease Prevention and Health Promotion, 2017), the RIDOH proposed an amendment to the current health care worker health screening requirements (Kim et al., 2014). The document Influenza Vaccine: A Regulatory Mandate in Rhode Island (Keough, 2014) described changes made to the regulations on immunization, testing, and health screenings for health care workers in Rhode Island. In 2012, an amendment to the state regulation was presented to reflect the ACIP's recommendation to mandate that health care workers receive the influenza vaccination annually or wear a mask when providing patient care. A public hearing was held to allow for arguments from various perspectives. Supporters of the influenza vaccine mandate argued that it is a health care worker's duty to protect their patients from infectious diseases, especially for those who are most vulnerable and those individuals where efficacy of the vaccine may be poor. Opponents of the change cited autonomy of nurse's rights, vaccine safety issues, and lack of vaccine efficacy (Keough, 2014). On October 23, 2012, Rhode Island 
became the first state in the nation to mandate that all health care workers receive influenza vaccination annually when providing direct care to patients (Keough, 2014).

Influenza vaccination coverage among health care workers was evaluated during the 2013-2014 influenza season in the state of Rhode Island as described in an article by Kim, Raymond, Washburn and Capelli (2014). The amendment required all health care workers to receive influenza vaccination or provide proof of a medical exemption or a signed declination statement to their health care facility not later than December $15^{\text {th }}$ each year beginning in 2012. Those who did not receive influenza vaccination would be required to wear a surgical mask during direct patient contact once influenza had been declared widespread by the RIDOH. Health care facilities are required to report health care worker vaccination data to the RIDOH at the end of the influenza season each spring. The authors found an increase in reporting by LTCF. In $2013-2014,87.7 \%$ of health care facilities reported their health care worker vaccination data as compared to the 2013-2014 and 2011-2012 influenza season where only 59.0\% and 26.9\% had reported. The data collected not only demonstrated an increase in reporting, it also showed an increase in health care worker vaccination. Vaccination of health care workers increased from $69.7 \%$ during the $2011-2012$ influenza season to $87.2 \%$ during the $2012-2013$ season and $88.1 \%$ during the $2013-2014$ season (Kim et al., 2014). The trend of increasing influenza vaccination rates for health care workers has brought the state close to the Healthy People 2020 goal of $90 \%$.

A study by Babcock, Gemeinhart, Jones, Dunagan, and Woeltje (2010) suggests that mandatory influenza vaccination of health care workers is the most effective method of increasing influenza vaccination rates. The setting used for this study was a large 
Midwestern health care facility with 23,000 employees. As part of their initiative, influenza vaccination was made a condition of employment. The vaccine was made available at no cost and medical or religious declinations were made available.

Individuals who did not receive the vaccine or demonstrate exemption were suspended without pay. The results of this study showed $98.4 \%$ of health care workers were vaccinated. This was an increase in vaccination rates by $43.4 \%$ in 2008 , compared with rates in 2006, which were 54\% (Babcock et al., 2010).

\section{Influenza Vaccination of Health Care Workers: Should it be Mandatory?}

Mandatory vaccination of health care workers continues to be controversial and

widely studied. A systematic review by Cortes-Penfield (2014) examined vaccination of health care workers as the new standard of care and asserted that health care workers have an ethical obligation to protect those for whom they care. The author cites promoting the acts of beneficence and non-maleficence in a way that promotes the patient's well-being (Cortes-Penfield, 2014). He concluded that it is the ethical responsibility of the health care workers to vaccinate themselves against influenza, and it is the responsibility of the health care facility to ensure and enforce influenza vaccination among its staff with the goal of reducing morbidity and mortality while reducing nosocomial infection.

A study by Music (2011) also aims to explore arguments for health care worker vaccination and initiatives. The author stated that research has shown that health care workers could potentially transmit influenza to those for whom they are providing care, and influenza vaccination is the best method to protect patients (Music, 2011). Many professional organizations in the United States such as the American Academy of 
Pediatrics (2010), the Association for Professionals in Infection Control and Epidemiology (2011), the Infectious Disease Society of America (2010), the National Patient Safety Foundation (2009), and the Society for Health care Epidemiology of America (2010) have put forth policies stating that increased influenza vaccine uptake can be achieved by presenting mandatory influenza vaccination as a patient safety measure.

\section{Influenza Vaccination and its Effect on Long-Term Care Facility Residents}

Evidence has clearly demonstrated the importance of health care worker influenza vaccination, especially for people living in long-term care facilities (CDC 2017c). Mandatory influenza vaccination programs have significantly increased the number of heath care workers who have received annual vaccination (Kim et al., 2014). In a randomized controlled study, Potter et al. (1997) hypothesized that vaccination of health care workers reduced the mortality of elderly patients in LTCF. The authors were able to demonstrate a reduction in patient mortality from $17 \%$ to $10 \%$, and reduction was associated with health care worker vaccination. They further noted that vaccination of patients, however, was not associated with effects on mortality (Potter et al., 1997).

Another study by Wendelboe, Avery, Andrade, Baumbach, and Landen (2011) also assessed the importance of influenza vaccination of employees in LTCF. The authors investigated the benefit of vaccination in preventing influenza in patients living in LTCF. Data collected for this study included influenza cases confirmed by testing and clinical presentation of ILI. During this two-year study, 17 outbreaks of influenza were reported, 11 of which were laboratory confirmed influenza and six of which were defined as ILI. An outbreak was defined as a single case of influenza in a LTCF resident. The authors 
concluded that increased vaccination coverage of staff is associated with fewer outbreaks of laboratory confirmed influenza and ILI and prevention of influenza in LTCF (Wendelboe et al., 2011).

It is clear from this literature review that the influenza virus poses a lifethreatening concern for the most vulnerable populations, in particular individuals living in LTCF. It is also known that vaccination, although not perfect, is the best method of preventing influenza infection, as well as preventing nosocomial influenza infection. The RIDOH state mandate has been demonstrated to be effective in increasing the number of health care workers who receive influenza vaccination on an annual basis. 


\section{Theoretical Framework}

The theoretical framework used to guide this research of evaluating the impact of the influenza vaccination mandate for health care workers in the state of Rhode Island on ILI and influenza incidence in LTCF is the CDC's Health Impact Pyramid. Dr. Thomas Frieden, who was the acting Director for the CDC from 2009-2016, designed the Health Impact Pyramid. In 2010, he established a five-tiered pyramid to describe the different types of public health interventions and their relative impact on improving health outcomes. The five tiers address counseling and education, clinical interventions, long lasting protective interventions, changing the context to make individuals default decisions healthy, and social economic factors. The greatest health impact is achieved at the lower base of the pyramid requiring the least amount of individual effort (Friedan, 2010) and has the greatest public health impact.

Education for many years has been the primary focus to emphasize the importance of health care worker influenza vaccination to protect patients from life threatening complications of influenza. Although many health care workers may have been influenced by education related to the importance of influenza vaccination, others still did not adhere to the recommendations. Counseling and education is the fifth tier on the pyramid. While education is important, it is noted as the least effective intervention. Vaccines target the third tier, providing long-lasting protective interventions. They protect the health care worker, the patient, as well as those who are unable to be vaccinated due to contraindications. Mandating influenza vaccination targets the second tier, changing of context level of the pyramid, making vaccination the default choice by 
way of policy. This type of policy intervention can significantly change health outcomes in a way that individual education is unable to match.

The Health Impact Pyramid depicted in Figure 2 below illustrates the levels of intervention with those at the base of the pyramid having comparatively greater impact on the population as compared with those at the peak.

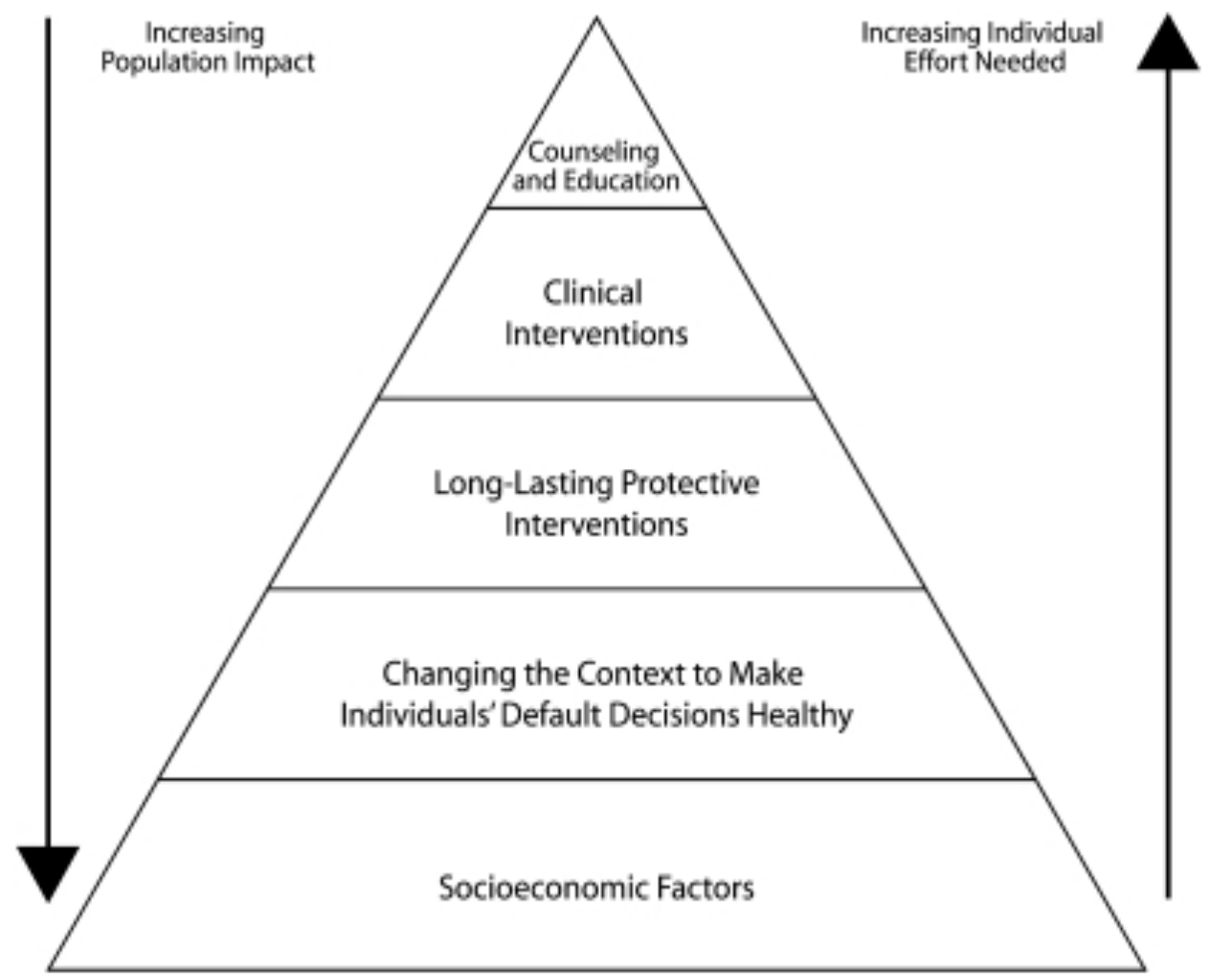

Figure 2. Health Impact Pyramid (Friedan, 2010). 


\section{Method}

\section{Purpose}

The purpose of this study was to evaluate the impact of the influenza vaccination mandate for health care workers in the state of Rhode Island on ILI and influenza related hospitalizations and deaths in individuals living in LTCF in Rhode Island. Currently, no evaluation on the impact of this policy on this population has been conducted.

\section{Sample/Site}

Data related to influenza in long-term care facilities reported to the Rhode Island State Health Lab and CAIDE from 2010-2017 was analyzed. Data for each of these years included the following: the number of reported ILI and laboratory confirmed influenza cases in LTCF, the number of hospitalizations related to influenza of individuals living in LTCF, and the number of influenza associated deaths of individuals living in LTCF. ILI data, considered to be an essential surveillance indicator, will be analyzed to estimate influenza incidence. Outcome measures such as ILI cases along with hospitalizations and deaths will offer a comprehensive view of influenza status within the state of Rhode Island.

\section{Design}

This study design was a secondary data analysis utilizing existing data from the RIDOH influenza seasons from 2010-2017. Data evaluated included the number of reported ILI and laboratory confirmed influenza cases, hospitalizations, and deaths associated with influenza in LTCF.

\section{Procedures}


A letter of agreement was obtained from the RIDOH demonstrating approval of the project (see Appendix A). IRB approval was obtained from Rhode Island College (Appendix B). Secondary data collection was conducted using surveillance data collected from sentinel sites by the RIDOH who have reported ILI, laboratory confirmed influenza, and hospitalizations and deaths associated with influenza. All data collected from the RIDOH was publicly accessible health data. The number of residents living in LTCF in Rhode Island during the periods analyzed was retrieved from the Centers for Medicare and Medicaid Services (CMS) website.

\section{Analysis}

Data was collected and stored using an excel spreadsheet and was analyzed from 2010-2017 to assess a negative relationship between policy implementation and influenza in residents of LTCF. The analysis will determine if there is an association between the implementation of the mandatory health care worker influenza vaccination mandates in the state of Rhode Island and the number of ILI, confirmed influenza cases, and hospitalizations and deaths from influenza in LTCF. 


\section{Results}

Public health data from the RIDOH was used to identify cases of ILI, confirmed influenza, hospitalizations, and deaths associated with influenza in LCTF in Rhode Island. Data was also collected from the CMS website to identify the number of residents living in LTCF.

Data from the RIDOH included cases of ILI, confirmed influenza, hospitalization, and deaths associated with influenza in LTCF in the state of Rhode Island for each year beginning with 2010 through 2017. Data were analyzed for 2010 and 2011 prior to the implementation of the mandatory influenza vaccination of health care workers in October 2012. Data were also analyzed from 2012-2017 for the years after the mandate. The years also reflect when data was available from the RIDOH. Data collected from the CMS website reflect the number of individuals living in LTCF for the calendar year.

Facilities included as LTCF include pediatric and adult rehabilitation. Using CMS data, the number of individuals living in LTCF was used to calculate the attack rate per 1000 residents that had ILI and laboratory confirmed influenza, hospitalizations, and deaths associated with influenza. Table 1 presents the number of cases and rate per 1000 for ILI, influenza related hospitalizations, and influenza related deaths for 2010-2017. Table 1

Rhode Island Influenza Like Illness (ILI), Influenza Related Hospitalizations, and Deaths Related to Influenza for LTCF Residents 2010-2017

\begin{tabular}{|c|c|c|c|c|c|c|c|c|}
\hline Year & \#of Residents & ILI & $\#$ of cases & Rate/1000 & \# of Hospitalizations & Hosp Rate/1000 & \# of Deaths & Death Rate/1000 \\
\hline 2010 & 7134 & 2010-2011 & 347 & 48.640 & Unknown & Unknown & 14 & 1.962 \\
\hline 2011 & 7608 & 2011-2012 & 57 & 7.492 & 0 & 0 & 2 & 0.263 \\
\hline 2012 & 7761 & $2012-2013$ & 572 & 73.702 & 53 & 6.829 & 19 & 2.448 \\
\hline 2013 & 8012 & 2013-2014 & 417 & 52.047 & 23 & 2.871 & 9 & 1.123 \\
\hline 2014 & 8012 & 2014-2015 & 1208 & 150.774 & 133 & 16.600 & 28 & 3.495 \\
\hline 2015 & 7973 & 2015-2016 & 148 & 18.563 & 15 & 1.881 & 2 & 0.251 \\
\hline 2016 & 7838 & 2016-2017 & 432 & 55.116 & 129 & 16.458 & 6 & 0.766 \\
\hline
\end{tabular}


Figures 3, 4, and 5 demonstrate the rate per 1000 residents of LTC facilities from 20102017 for ILI, hospitalizations, and deaths, respectively.

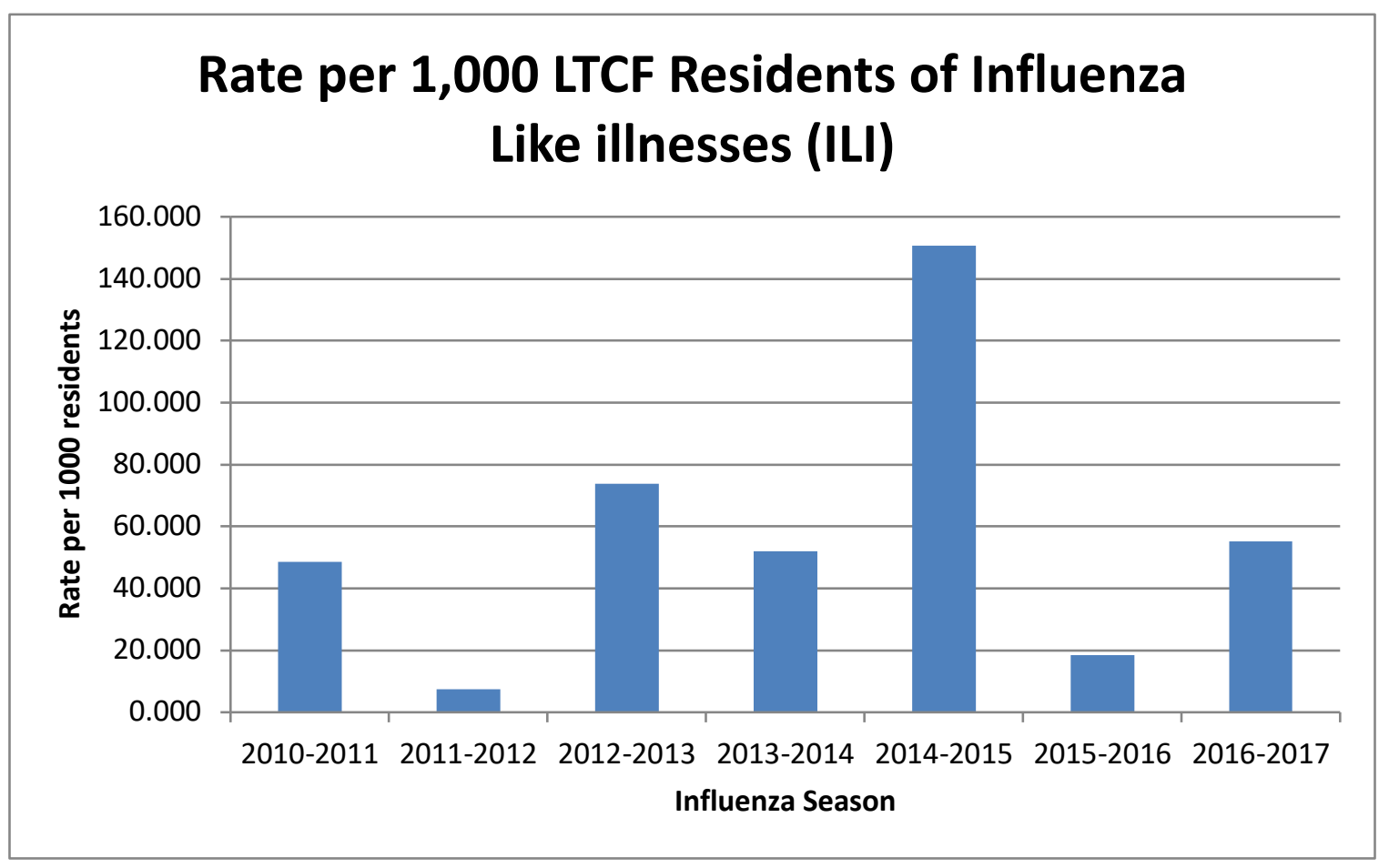

Figure 3. Rate of Influenza Like Illness (ILI) Among LTCF Residents 


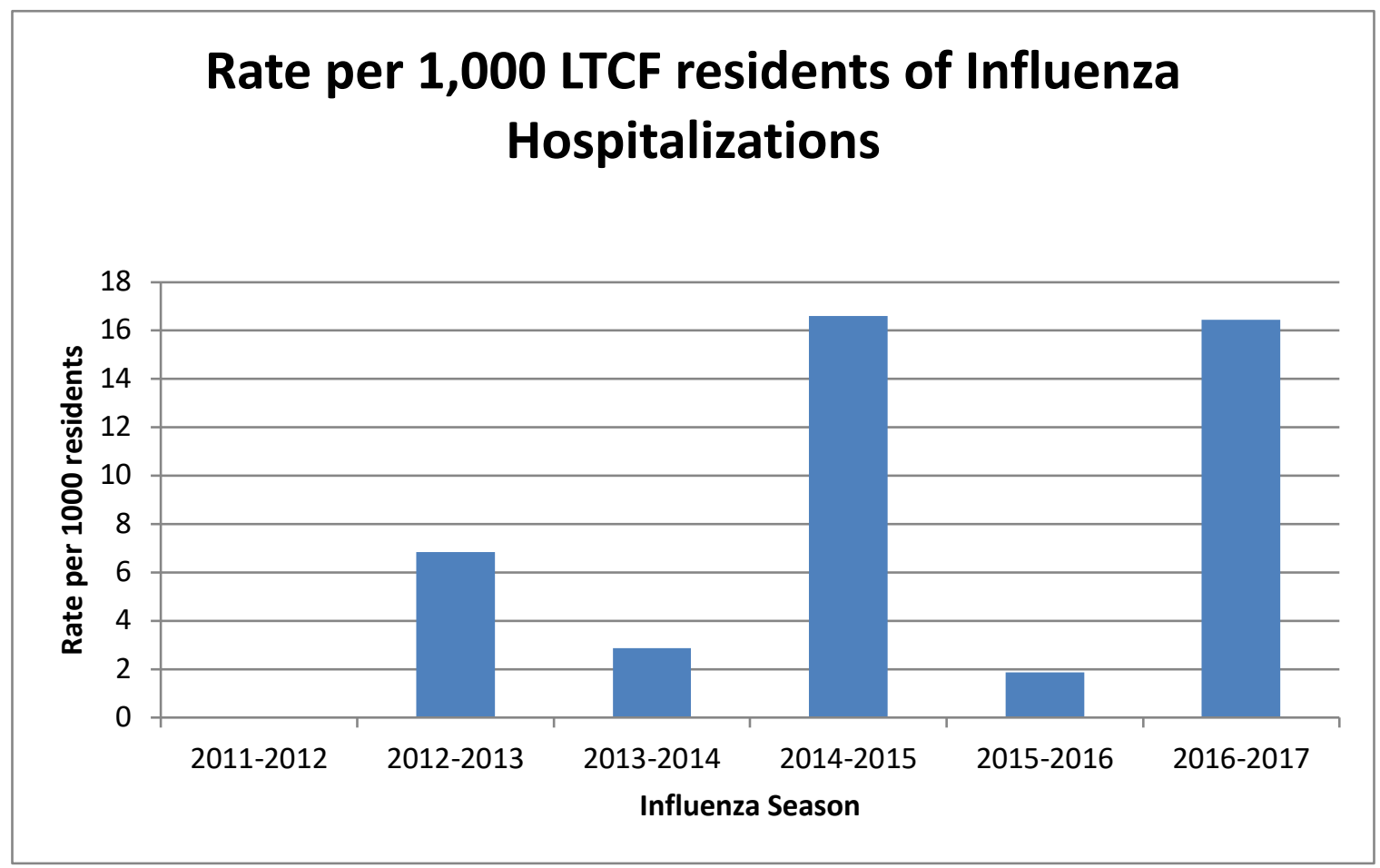

Figure 4. Rate of Influenza Associated Hospitalizations Among LTCF Residents

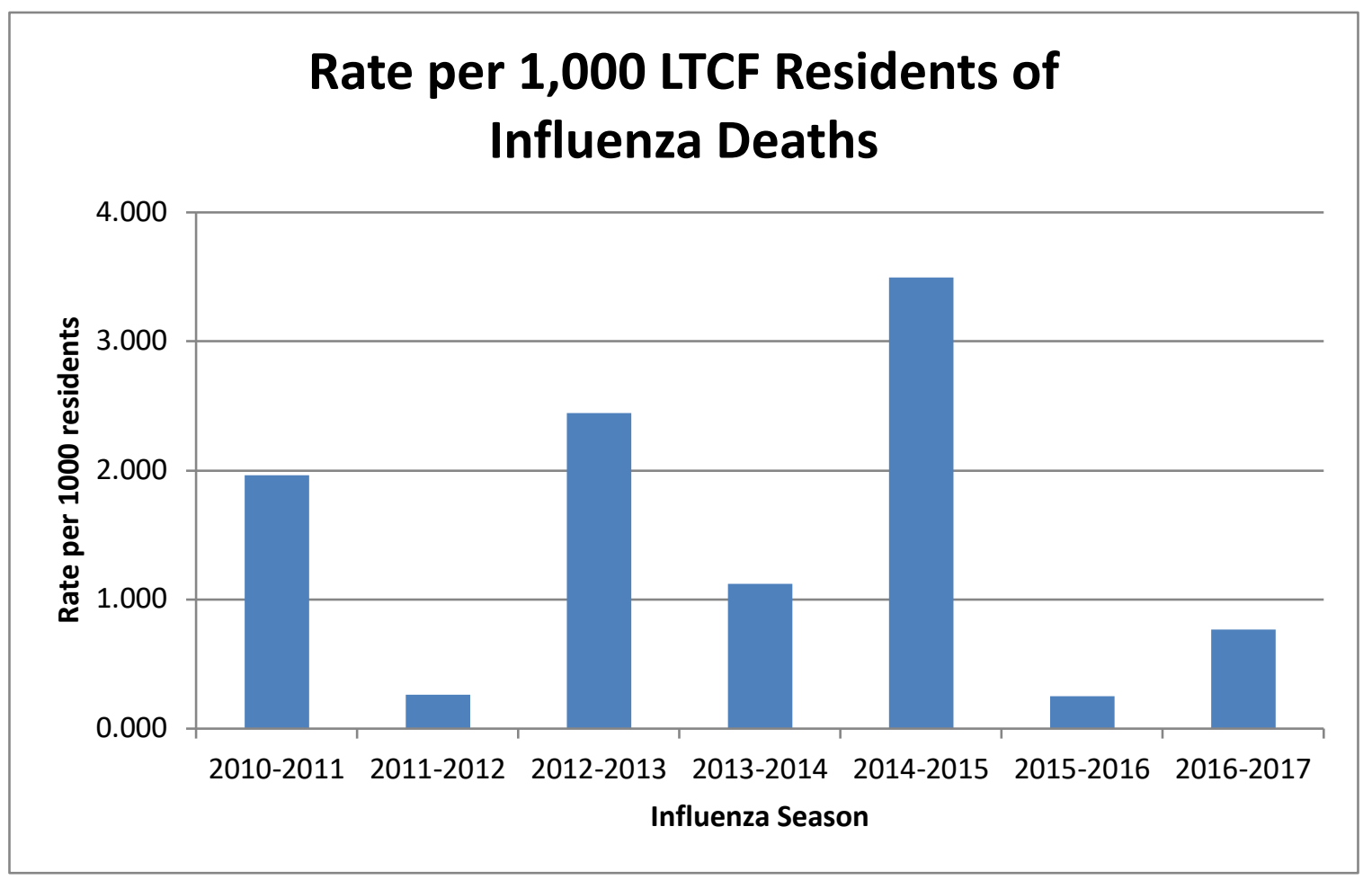

Figure 5. Rate of Influenza Related Deaths Among LTCF Residents 
Prior to the mandate, during the 2010-2011influenza season, the attack rate for ILI and confirmed influenza was 48.64 per 1000 . The death rate for that time period was 1.96 per 1000 for deaths. The number of hospitalizations is unknown due to reporting issues. For the 2011-2012 season, the ILI attack rate was noted at 7.49 per 1000 individuals for ILI and confirmed influenza and 0.26 per 1000 for deaths.

Data during the 2012-2013 influenza season, after the mandatory influenza regulation for health care workers was established, demonstrated an increased rate of 73.0 per 1000 for ILI and confirmed influenza. Hospitalizations for this time period were 6.82 per 1000. Deaths for this time period were also higher at 2.44 per 1000. A lower rate of ILI and hospitalizations was seen in the 2013-2014 season as compared with the previous influenza season with an attack rate of 52.04 per 1000 noted for ILI and confirmed influenza, 2.87 per 1000 for hospitalizations, and 1.12 per 1000 for deaths.

The 2014-2015 influenza season demonstrated the highest rate for ILI and confirmed cases with a rate of 150.77 per 1000 individuals, 16.60 per 1000 for hospitalizations, and 3.49 per 1000 for deaths associated with influenza. A rate of 18.56 per 1000 for ILI and confirmed influenza, 1.88 per 1000 for hospitalizations, and 0.25 per 1000 deaths was noted during the 2015-2016 season, much lower than the previous season. During the 2016-2017 influenza season, a rate of 55.11 per 1000 ILI and confirmed influenza cases, 16.45 per 1000 hospitalizations, and 0.76 per 1000 deaths. This influenza season demonstrates a spike in ILI and hospitalization, and death rates as compared with the previous year.

One critical variable impacting influenza ILI, hospitalization, and death rates is the effectiveness of the influenza vaccine. An assessment of the overall effectiveness of 
the influenza vaccine is important to consider when analyzing data for the 2010-2016 influenza seasons. The adjusted vaccine effectiveness estimates for 2005-2018 are presented in Appendix C. According to the CDC, the 2010-2011 influenza season was a less severe season in comparison to the previous two seasons and the most reported virus was influenza A (H3N2) which often affects individuals 65 years of age or older (CDC, 2011a). During this influenza season the adjusted overall vaccine effectiveness was $60 \%$. The CDC noted H3N2 as the predominant strain during the 2011-2012 influenza season. In comparison to other influenza seasons, a new record was set for the lowest and shortest peak of ILI with an overall adjusted vaccination effectiveness of $47 \%$ (CDC, 2012). The 2012-2013 influenza season was characterized by the CDC as a moderately severe season with a high number of outpatient visits for ILI reported and high rates of hospitalizations among people 65 years and older (CDC, 2013). H3N2 was the predominant strain with an overall adjusted vaccine effectiveness of 49\% (CDC, 2013). The 2013-2014 season reported severe influenza illness among young and middle-aged adults. The prevalent strain for this influenza season was H1N1, with an overall adjusted vaccine effectiveness of $52 \%$ (CDC, 2014).

The largest attack rate was noted during the 2014-2015 influenza season in which H3N2 predominated. This season also had the lowest overall adjusted vaccine effectiveness of 19\% (CDC, 2015). This influenza season was moderately severe and was a severe one for individuals 65 years and older and the lowest vaccine efficacy for all influenza seasons evaluated. This corresponds with the high rates of ILI, hospitalizations, and deaths seen in Figures 3, 4, and 5 for the 2014-2015 season. The influenza season for 
2015-2016 was a milder season with an adjusted vaccine effectiveness of 48\% (CDC, 2016a).

Although H3N2 was predominate earlier in the season, H1N1 was most common later in the season (CDC, 2016a). For the 2016-2017 influenza season, H3N2 was the most prevalent strain. This was a moderate influenza season with high outpatient illness and influenza-associated hospitalizations among individuals 65 or older (Burns et al., 2017). The overall adjusted vaccine effectiveness was $40 \%$ (CDC, 2017b).

Based on this data, it is unclear whether the RIDOH's mandatory influenza vaccination of health care workers had an impact on decreasing the number of cases of ILI and confirmed influenza, hospitalizations and deaths associated with influenza in the short-term. Confounding factors such as vaccine efficacy and the circulating strains of influenza virus made it difficult to ascertain the total impact this policy may have had on this vulnerable population. 


\section{Summary and Conclusions}

\section{Limitations}

Some limitations of the study influenced the outcomes. These include: virulence of influenza strain during various influenza seasons, the effectiveness of the annual vaccine, the number of health care workers vaccinated, the number of residents vaccinated, increased reporting by LTCF following the mandate, change in the RIDOH influenza data collection methods, CMS data which provided data for calendar years versus influenza season, and unknown vaccination status of visitors within the LTCF.

For example, several of the years evaluated demonstrated decreased efficacy of the influenza vaccine due to changes in the circulating virus strain during the influenza season. It was also unknown which influenza vaccine residents received, quadrivalent standard dose or trivalent high dose, which may impact the data. In addition, data collection methods by the RIDOH varied throughout the years evaluated. More recent precise measuring of influenza and increased compliance with reporting in LTCF may have created the appearance of increased rates when in actuality the data was just better captured.

In addition, the project was a secondary data analysis which attempted to evaluate if there was a decrease in the number of ILI, laboratory confirmed influenza, hospitalizations and deaths associated with influenza since the influenza vaccination mandate was implemented in 2012. While the policy may not appear to impact influenza vaccination rates initially, it remains unclear if the policy will impact influenza related outcomes in the long-term. Evaluation over a greater number of years from mandated vaccination may improve the quality of the findings. Long-term data may demonstrate 
trends of decreasing ILI, hospitalizations, and deaths from influenza which are not apparent with a data over the short period evaluated in this study.

\section{Summary}

The purpose of this project was to determine the impact of the RIDOH mandatory influenza vaccination of health care workers on ILI, laboratory confirmed influenza, hospitalizations, and deaths associated with influenza among LTCF residents. Data was analyzed for influenza seasons starting in 2010 through 2017. Data was analyzed for the influenza seasons prior to the mandate, during the mandate, and after the mandate. The results indicate a need for further study including longitudinal and experimental research. While the policy over time may have increased influenza vaccination rates among health care workers, it remains unclear if the policy will impact influenza related outcomes in the long term. At this time, it is unclear whether the mandate had an impact on residents in LTCF.

\section{Conclusions}

It is clear that, although imperfect due to variable efficacy, vaccination is the best method of protection, particularly for those who are most vulnerable. Health care workers have an ethical responsibility to do no harm which includes protecting patients from infectious diseases. Vaccination remains one of the most effective methods of primary prevention towards protecting the population from serious illness and death. The results of this project demonstrates a need for further long-term study to evaluate the impact of influenza vaccination on influenza incidence in LTCF residents. 


\section{Recommendations and Implications for Advanced Nursing Practice}

Implications for practice, education, research, and policy are clear. The advanced practice nurse can be instrumental in change in all practice settings including long-term

care. It is the role of the advanced practice nurse to collaborate with community practice partners to ensure the health and safety of our populations, especially those who are most vulnerable, including the young, elderly, and those who are immunocompromised. Advanced practice nurses should provide education for health care workers and the public on the evidence based need for vaccination of workers, residents, and the larger population to provide herd immunity and reduce risk. Through education, advanced practice nurses can begin changing the culture of nursing to understand the safety element vaccination has toward protecting patients.

Recommendations for research include conducting higher level epidemiological research including cohort and experimental studies to evaluate the effectiveness of health care worker vaccination in preventing negative influenza outcomes in LTCF residents and expanding the findings to a variety of practice sites. In addition, correlational analysis in the future when the policy has been in effect over the long term may provide information about the impact of this policy. Advance practice nurses must advocate for public health and safety policies through leadership in state and local public health agencies. Through collaboration with stakeholders, they can demonstrate the need for expansion of influenza vaccination mandates to other states. 


\section{References}

American Academy of Pediatrics. (2010). Policy statement - recommendation for mandatory influenza immunization of all health care personnel. Pediatrics, 126, 809-815. Retrieved from http://pediatrics.aappublications.org/content/early/2010/09/13/peds.2010-2376

Association for Professionals in Infection Control and Epidemiology. (2011). APIC calls for mandatory annual flu immunization of health care workers. Retrieved from https://apic.org/For-Media/News-Releases/Article?id=f46a68b0-4916-440c-8f88$713 \mathrm{be} 4595521$

Babcock, M., Gemeinhart, N., Jones, M., Dunagan, W., \& Woeltje, K. (2010). Mandatory influenza vaccination of health care workers: Translating policy to practice. Clinical Infectious Diseases, 50, 459-464. Doi:10.1086/650752

Burns, A. L., Shuford, S. H., Brady, D., Calcione, C., Quilliam, D. N., Fulton, J. P., \& Bandy, U. (2017). Influenza epidemiology report: 2016-2017 Rhode Island surveillance report. Retrieved from www.health.ri.gov/publications/surveillance/2017/Influenza.pdf

Centers for Disease Control and Prevention. (2011a). Update: Influenza activity_-United States, 2010-11 season, and composition of the 2011-12 influenza vaccine. MMWR. 60(21):705-712. Retrieved from https://www.cdc.gov/mmwr/preview/mmwrhtml/mm6021a5.htm

Centers for Disease Control and Prevention. (2011b). Immunization of health-care personnel: Recommendations of the advisory committee on immunization 
practices (ACIP). MMWR. 60(RR07):1-45. Retrieved from

https://www.cdc.gov/mmwr/preview/mmwrhtml//rr6007a1.htm

Centers for Disease Control and Prevention. (2012). Update: Influenza activity—United States, 2011-12 season, and composition of the 2012-12 influenza vaccine. MMWR. 61(22):414-420. Retrieved from https://www.cdc.gov/mmwr/preview/mmwrhtml/mm6122a4.htm

Centers for Disease Control and Prevention. (2013). Update: Influenza activity—United States, 2012-13 season, and composition of the 2013-14 influenza vaccine. MMWR. 62(23):473-479. Retrieved from https://www.cdc.gov/mmwr/preview/mmwrhtml/mm6223a5.htm Centers for Disease Control and Prevention. (2014). Update: Influenza activity—United States, 2013-14 season, and composition of the 2014-15 influenza vaccine. MMWR. 63(22):483-490. Retrieved from https://www.cdc.gov/mmwr/preview/mmwrhtml/mm6322a2.htm Centers for Disease Control and Prevention. (2015). Update: Influenza activity—United States, 2014-15 season, and composition of the 2015-16 influenza vaccine. MMWR. 64(21):583-590. Retrieved from https://www.cdc.gov/mmwr/preview/mmwrhtml/mm6421a5.htm Centers for Disease Control and Prevention. (2016a). Update: Influenza activity—United States, 2015-16 season, and composition of the 2016-17 influenza vaccine. MMWR. 65(22):576-575. Retrieved from https://www.cdc.gov/mmwr/volumes/65/wr/mm6522a3.htm 
Centers for Disease Control and Prevention. (2016b). Influenza vaccination coverage among health care personnel - United States, 2015-16-influenza season. $M M W R$ 65(38): 1026-1031. Retrieved from https://www.cdc.gov/mmwr/volumes/65/wr/mm6538a2.htm

Centers for Disease Control and Prevention. (2016c). Overview of influenza surveillance in the United States. Retrieved from https://www.cdc.gov/flu/weekly/overview.htm

Centers for Disease Control and Prevention. (2016d). Seasonal influenza: Flu basics. Retrieved from https://www.cdc.gov/flu/about/disease/index.htm

Centers for Disease Control and Prevention. (2017a). Disease burden of influenza. Retrieved from https://www.cdc.gov/flu/about/disease/burden.htm Centers for Disease Control and Prevention. (2017b). Influenza activity in the United States during the 2016-17 season and composition of the 2017-18 influenza vaccine. $M M W R$. 66(25):668-676. Retrieved from https://www.cdc.gov/mmwr/volumes/66/wr/mm6625a3.htm

Centers for Disease Control and Prevention. (2017c). The importance of influenza vaccination for health care personnel in long-term care. Retrieved from https://www.cdc.gov/flu/toolkit/long-term-care/importance.htm

Centers for Disease Control and Prevention. (2017d). Vaccination laws. Retrieved from https://www.cdc.gov/phlp/publications/topic/vaccinationlaws.html

Center for Disease Control and Prevention. (2017e). Vaccination recommendations of the ACIP. Retrieved from https://www.cdc.gov/vaccines/hcp/acip-recs/vaccspecific/flu.html 
Centers for Disease Control and Prevention. (2018). Seasonal influenza vaccine effectiveness, 2005-2018. Retrieved from https://www.cdc.gov/flu/professionals/vaccination/effectiveness-studies.htm

Centers for Medicare \& Medicaid Services. (2018). Nursing home datasets [Data file]. Retrieved from https://data.medicare.gov/data/nursing-home-compare

Cortes-Penfield, N. (2014). Mandatory influenza vaccination for health care workers as the new standard of care: A matter of patient safety and nonmaleficent practice. American Journal of Public Health, 104(11), 2060-2065.

Doi:10.2105/AJPH.2013.201514

Eibach, D., Casalegno, J. S., Bouscambert, M., Benet, T., Regis, C., Comte, B., ... Lina, B. (2014). Routes of transmission during a nosocomial influenza A (H3N2) outbreak among geriatric patients and health care workers. Journal of Hospital Infection, 86, 88-193. http://dx.doi.org/10.1016/j.jhin.2013.11.009

Frenzel, E., Chemaly, R. F., Sriza-Heredia, E., Jiang, Y., Shah, D. P., Thomas, G., ... Raad, I. (2016). Association of increased influenza vaccination in health care workers with a reduction in nosocomial influenza infections in cancer patients. American Journal of Infection Control, 44, 1016-1021. Doi:

10.1016/j.ajic.2016.03.24

Frieden, T. R. (2010). A framework for public health action: The health impact pyramid. American Journal of Public Health, 100(4), 590-595. Doi:

10.2105/AJPH.2009.185652

Infectious Disease Society of America. (2010). IDSA policy on mandatory immunization of health care workers against seasonal and pandemic influenza. Retrieved from 
https:// http://www.idsociety.org/HCWimmunization

Inweregbu, K., Dave, J., \& Pettard, A. (2005). Nosocomial infections. Continuing Education in Anesthesia, Critical Care \& Pain, 5, 14-16. Doi:

10.1093/bjaceaccp/mki006

Keough, L. (2014). Influenza vaccine: A regulatory mandate in Rhode Island. Journal of Nursing Regulation, 4(4), 36-39. Doi:10.1016/S2155-8256(15)30107-1

Kim, H., Dube, D., Kalayil, E. J., Lindley, M. C., Paiva, K. A., \& Raymond, P. (2014). Evaluation of the impact of the 2012 Rhode Island health care worker influenza vaccination regulations: Implementing process and vaccination coverage. Journal of Public Health Management and Practice, 00(00), 1-9.

Doi:10.1097/PHH.0000000000000128

Kim, H., Raymond, P., Washburn, T., \& Capelli, D. (2014). Influenza vaccination coverage among health care workers during the 2013-14 influenza season in Rhode Island. Rhode Island Medical Journal, 97(10), 60-61. Retrieved from http://www.rimed.org/rimedicaljournal/2014/10/2014-10-60-health-kim.pdf Marshall, R. J., Tetu-Mouradjian, L. M., \& Fulton, J. P. (2010). Increased annual influenza vaccinations among health care workers in Rhode Island: A social marketing approach. Medicine \& Health/Rhode Island, 93(9), 271-278. Retrieved from http://www.rimed.org/medhealthri/2010-09/2010-09-271.pdf

Molinari, N. A., Ortega-Sanchez, I. R., Messonnier, M. L., Thompson, W. W., Wortley, P. M., Weintraub, E., \& Bridges, C. B. (2007). The annual impact of seasonal influenza in the US: Measuring disease burden and costs. Elsevier Vaccine, 25, 5086-5096. Doi: 10.1016/j.vaccine.2007.03.046 
Music, T. (2011). Protecting patients, protecting health care workers: a review of the role of influenza vaccination. International Nursing Review, 59(2). Doi: $10.1111 / \mathrm{j} .1466-7657.2011 .00961 . x$

National Patient Safety Foundation. (2015). National patient safety foundation supports mandatory flu vaccinations for health care workers. Retrieved from http://www.npsf.org/news/259784/National-Patient-Safety-Foundation-SupportsMandatory-Flu-Vaccine-for-Health-Care-Workers.htm

Office of Disease Prevention and Health Promotion. (2017). Healthy People 2020. Retrieved from https://www.healthypeople.gov/node/6361/data_details

Pless, A., McLennan, S. R., Nicca, D., \& Elger, B. S. (2017). Reasons why nurses decline influenza vaccination: a qualitative study. BMC Nursing, 16(20). Doi:10.1186/s12912-017-0215-5

Potter, J., Stott, D. J., Roberts, M. A., Elder, A. G., O’Donnell, B., Knight, P. V., \& Carman, W. F. (1997). Influenza vaccination of health care worker in long-termcare hospitals reduces the mortality of elderly patients. The Journal of Infectious Diseases, 175(1). Doi: 10.1093/infdis/175.1.1

Rhode Island Department of Health. (2012). HEALTH adopts regulations to require flu shots for health care workers in Rhode Island [Press release]. Retrieved from http://www.ri.gov/press/view/17589

State of Rhode Island and Providence Plantations Department of Health. (2012). Rules and Regulations Pertaining to Immunization, Testing, and Health Screening for Health Care Workers [R23-17-HCW]. Retrieved from sos.ri.gov/documents/archives/regdocs/released/pdf/DOH/7083.pdf 
Society for Health care Epidemiology of America. (2010). SHEA position paper: Influenza vaccination of health care personnel. Infection Control and Hospital Epidemiology, 31(10), 987-995.

The College of Physicians of Philadelphia. (2018). The history of vaccines. Retrieved from http:/www.historyofvaccines.org

Wendelboe, A. M., Avery, C., Andrade, B., Baumbach, J., \& Landen, M. G. (2011). Importance of employee vaccination against influenza in preventing cases in longterm care facilities. Infection Control and Hospital Epidemiology, 32(10). Doi: $10.1086 / 661916$

Willis, B. C., \& Wortley, P. (2007). Nurses' attitudes and beliefs about influenza and the influenza vaccine: A summary of focus groups in Alabama and Michigan. American Journal of Infection Control, 35, 20-24. Doi:10.1016/j.ajic.2006.07.009 


\section{Appendix A}

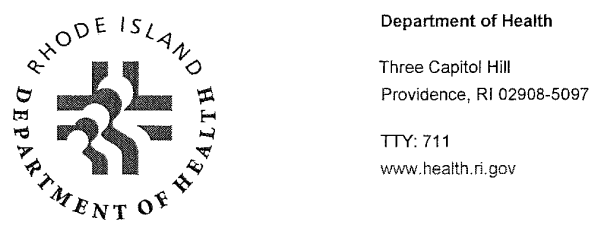

Diane S. Brady, MS, RN

Public Health Nurse Consultant

Rhode Island Department of Health

Center for Acute Infectious Disease

And Epidemiology

Room 106, 3 Capitol Hill

Providence, RI 02908

Rhode Island College

Institutional Research Board

600 Mount Pleasant Avenue

Providence, RI 02908

September 20, 2017

To whom it may concern:

This letter is provided as agreement to serve as a collaborating agency on RIC

School of Nursing graduate student Wendy Chicoine's masters project titled, The Impact of

Rhode Island's Mandatory Healthcare Worker Influenza Vaccination on Influenza Incidence in

Long Term Care Facility Residents. This project will involve the analysis of influenza data

collected by the Rhode Island Department of Health. The student will be supported to access this data through the Center for Acute Infectious Disease Epidemiology at the Rhode Island

Department of Health. The Rhode Island Department of Health is supportive of this project and will facilitate the student's access to the data.

Sincerely,

Nuane \& Brody MS, RN

Diane S. Brady, MS, RN

Public Health Nurse Consultant

Rhode Island Department of Health

Center for Acute Infectious Disease Epidemiology 


\section{Appendix B}

-a IRB: \#1718-1531 (Costello, Joanne) exempt

N NoReplyRIC_Elements@topazti.net <NoReplyRIC_Elements@topazti.net>

irb@ric.edu; jcostello@ric.edu; wchicoine_2162@email.ric.edu

Tuesday, October 31, 2017 at 12:45 PM

Show Details

Greetings,

The proposal for the project referenced below has been determined to be exempt by the Institutional Review Board (IRB).

Project title: The Impact of Rhode Island's Mandatory Heathcare Worker Influenza Vaccination on Influenza in Long-Term Care Facility Residents

\#: 1718-1531

Type of review: Exempt

Proposal type: Original

Principle Investigator: Costello, Joanne

Fees received: 1. No fees -- RIC supervised or sponsored

Funding status:

Date: $10 / 31 / 2017$

Expiration date: $10 / 31 / 2020$

A request to renew this protocol must be received by $10 / 3 / 2020$.

Click here to access the protocol: https://ric.topazti.net/RIC/SL/Default. aspx?linkParms=NPakQNfZcenU5zIgSrogplo\%3d\%3d

This protocol has been reviewed to determine that it met the criteria for an exemption. An exemption is not the same as an approval. Researchers are encouraged to adhere to the same ethical standards as non exempt research.

Changes to the protocol methods may change the approval status and should be reviewed by the IRB prior to implementation.

You must keep all research data and consent documents within your possession in a secured location for at least three (3) years after the completion of the study, including publications or presentations of any reports.

Do not reply to this "RIC_Elements" email address because it will not be received by the IRB. Send all correspondence to IRBQric.edu.

Best Regards,

Cindy Padula, Ph.D.

Professor

Chair, IRB

Rhode Island College

IRBQric.edu 


\section{Appendix C}

Table. Adjusted Vaccine Effectiveness Estimates for Influenza Seasons from 2005-2018

\begin{tabular}{|c|c|c|c|c|c|}
\hline Influenza Season ${ }^{\dagger}$ & Reference & Study Site(s) & No. of Patients ${ }^{\ddagger}$ & Adjusted Overall VE (\%) & $95 \% \mathrm{Cl}$ \\
\hline $2004-05$ & Belongia 2009 & WI & 762 & 10 & $-36,40$ \\
\hline $2005 \cdot 06$ & Belongia 2009 & WI & 346 & 21 & $-52,59$ \\
\hline $2006-07$ & Belongia 2009 & WI & 871 & 52 & 22,70 \\
\hline $2007 \cdot 08$ & Belongia 2011 & WI & 1914 & 37 & 22,49 \\
\hline $2008-09$ & Unpublished & WI, MI, NY, TN & 6713 & 41 & 30,50 \\
\hline $2009-10$ & Griffin 2011 & WI, MI, NY, TN & 6757 & 56 & 23,75 \\
\hline $2010-11$ & 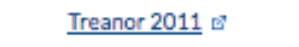 & WI, MI, NY, TN & 4757 & 60 & 53,66 \\
\hline $2011-12$ & Ohmit 2014 & WI, MI, PA, TX,WA & 4771 & 47 & 36,56 \\
\hline $2012-13$ & McLean 2014 & WI, MI, PA, TX,WA & 6452 & 49 & 43,55 \\
\hline 2013-14 & Gaglani 2016 口 & WI, MI, PA, TX,WA & 5999 & 52 & 44,59 \\
\hline $2014-15$ & Zimmerman 2016 & WI, MI, PA. TX,WA & 9311 & 19 & 10,27 \\
\hline $2015-16$ & Jackson 2017 & WI, MI, PA, TX,WA & 6879 & 48 & 41,55 \\
\hline $2016-17^{*}$ & Unpublished final estimates. & WI, MI, PA, TX,WA & 7410 & $40^{\circ}$ & $32,46^{\circ}$ \\
\hline $2017-18^{* *}$ & Flannery 2018 & WI, MI, PA, TX,WA & 4,562 & $36^{* *}$ & $27,44^{*}$ \\
\hline
\end{tabular}

"Interim 2016-2017 VE estimates (4/20/2016-4/9/2017) were presented to ACIP in June 2017 쥬 [743KB, 19 pages]

"*nterim early estimates may differ from final end-of-season estimates.

(CDC, 2018) 